\title{
CÁNCER DE CUELLO UTERINO: SOBREVIDA A 3 y 5 AÑOS EN HOSPITAL SAN JOSÉ
}

\author{
Pablo Sepúlveda V. 1,2, Fernando González C. 1,2, Cayetano Napolitano R. 1,2, \\ Enrique Roncone D. ${ }^{1,2}$, Gabriel Cavada C. ${ }^{3}$
}

1 Servicio y Departamento de Obstetricia y Ginecología, Unidad de Patología Cervical, Hospital San José. ${ }^{2}$ Universidad de Santiago de Chile. ${ }^{3}$ Escuela de Salud Pública, Universidad de Chile.

\section{RESUMEN}

Antecedentes: El cáncer cérvico uterino es la quinta causa de muerte por cáncer en la mujer chilena. Objetivo: Comparar entre agosto de 1999 y diciembre de 2002 la sobrevida por cáncer cérvico uterino según estadio clínico a 3 y 5 años diagnosticados en la Unidad de Patología Cervical del Hospital San José. Método: La sobrevida se calculó con la totalidad de casos de cáncer cérvico uterinos diagnosticados, su estadio y mortalidad a 3 y 5 años obtenidos a través del Registro Civil de Chile. Resultados: La distribución por estadio fue: $22,2 \%$ para estadio I, $21,3 \%$ para estadio II, $53,7 \%$ para estadio III y $2,7 \%$ para estadio IV. En estadio I la sobrevida a 3 años fue de $83 \%$ (IC: 61,5 - 93,4) y a 5 años de $78 \%$ (IC: $55,5-90,5$ ); en estadio II fue de $65 \%$ (IC: $42,4-80,8$ ) y de $60 \%$ (IC: $38,3-77,4$ ), respectivamente; en estadio III fue de $33 \%$ (IC: 21,6 - 45,5) y $29 \%$ (IC: 18,6 - 41,9), respectivamente; y en estadio IV la mortalidad a 3 y 5 años fue de $100 \%$ produciéndose el deceso durante los tres primeros meses desde su diagnóstico. Conclusión: La sobrevida se correlaciona directamente con el estadio clínico en el momento del diagnóstico, independiente de la edad de la paciente, debiendo aumentar la cobertura del tamizaje, mejorando los tiempos de confirmación diagnóstica y tratamiento óptimo, como también mejorando los sistemas de redes y registros.

\section{PALABRAS CLAVE: Cáncer cérvico uterino, estadio, sobrevida, Chile}

\section{SUMMARY}

Background: Cervical cancer is the fifth cause of death by cancer in Chilean women. Objectives: To compare between August 1999 and December 2002 the survival of cervical cancer according to a clinical stage in 3 and 5 years diagnosed in the Cervical Pathology Unit of San Jose Hospital. Methods: The overall survival rate was calculated with the totality of diagnosed cervical cancer, his stage and mortality in 3 and 5 years was obtained through the National Registry Office. Results: The distribution for stage was: $22.2 \%$ for stage I, $21.3 \%$ for stage II, $53.7 \%$ for stage III and $2.7 \%$ for stage IV. In stage I the survival at 3 years was $83 \%$ (Cl: 61.5 - 93.4), and at 5 years $78 \%$ (Cl: 55.5 - 90.5). In stage II the survival at 3 years was $65 \%$ (Cl: 42.4 - 80.8) and at 5 years $60 \%$ (Cl: $38.3-77.4)$. In stage III the survival at 3 years was $33 \%(\mathrm{Cl}: 21.6-45.5)$ and at 5 years $29 \%$ (Cl: $18.6-41.9)$. In stage IV the mortality at 3 and 5 years was $100 \%$ produced during the first 3 months from the diagnosis. Conclusions: The survival is directly correlative with the clinical stage in the moment of the diagnosis, independent of the age of the patients, by which it must increase the screening coverage, improving the times of diagnostic confirmation and optimum treatment, and also improving the network systems and registries. 


\section{INTRODUCCIÓN}

En el mundo anualmente se diagnostican alrededor de 500.000 casos de cáncer cérvico uterino (Cacu) invasor. Representa el $12 \%$ de todos los cánceres diagnosticados en mujeres (1). En Chile, es la quinta causa de muerte por cáncer en la mujer, luego de los cánceres de mama, gástrico, vesícula y vías biliares, y broncopulmonar, con una tasa de mortalidad de 8,5 por 100.000 mujeres (2). La tasa de años de vida potencialmente perdidos (AVPP) en la mujer por Cacu es de 129 por 100.000 mujeres. Ésta es la mayor tasa asignándole una trascendencia social y económica, ya que afecta a mujeres relativamente jóvenes y en edad reproductiva. Sin embargo, es el tumor maligno con mejores posibilidades de pesquisa y tratamiento $(3,4,5)$.

La mortalidad en menores de 25 años es casi inexistente y el aumento del riesgo de morir comienza a partir de los 35 años, incrementándose progresivamente. Es la primera causa de muerte por tumores malignos en mujeres entre los 30 a 54 años. Más del $80 \%$ de las muertes se produce en mujeres entre los 40 a 60 años. La incidencia anual estimada de cáncer invasor es de aproximadamente 18 por 100.000 mujeres. A través del Programa Nacional de Prevención de Cacu se ha visto una disminución en la mortalidad por este cáncer desde 24 por 100.000 mujeres en 1987 a 8,5 por 100.000 en $2003(2,3,4)$.

El Programa Nacional de Prevención de Cacu en Chile está enfocado al grupo de mujeres de entre 25 y 65 años. Se realiza control de Papanicolaou cada tres años con especial énfasis en mujeres mayores de 35 años y mujeres sin citologías previas. En relación a la cobertura, las últimas cifras demuestran un progresivo aumento alcanzando el $66 \%$ en 2001, sin llegar a los estándares óptimos de $80 \%$. La detección precoz y el tratamiento oportuno son los objetivos del Programa pretendiendo reducir en un $50 \%$ la mortalidad por Cacu, alcanzando coberturas de un $80 \%$ en mujeres beneficiarias de 25 a 64 años con citología cervical vigente $(5,7)$.

En relación a su pronóstico, el más importante es el estadio clínico. La supervivencia global de la paciente con $\mathrm{Cacu}$, tratada de forma adecuada, sería de $65 \%$ a los 5 años, pero las expectativas de vida varían según estadios clínicos de extensión. En el estadio la alcanzaría el $95 \%$, en el estadio lb de $81 \%$, en el estadio Ila de $66 \%$, en el estadio Ilb alrededor de $63 \%$, en estadio III entre 33 a $38 \%$ y finalmente en estadio IV entre 9 y $17 \%(8,9)$. Otros factores pronóstico son: tamaño tumoral, profundidad de la invasión, metástasis a ganglios linfáticos, presencia de células neoplásicas en espacios vas- culares y linfáticos y tipo histológico.

El objetivo del estudio es evaluar la sobrevida según estadio, a 3 y 5 años, de 108 mujeres con Cacu diagnosticados en el Hospital San José en el período agosto de 1999 a diciembre de 2002.

\section{MATERIAL Y MÉTODO}

Se realizó un análisis prospectivo histórico de todas las pacientes con Cacu diagnosticado en la Unidad de Patología Cervical del Servicio de Ginecología y Obstetricia del Hospital San José, desde agosto de 1999 a diciembre de 2002, por un período de 3 y 5 años con un total de 108 pacientes. Se revisó la ficha clínica de cada paciente registrando la edad, fecha de diagnóstico, tipo histológico y etapificación clínica. El Cacu fue confirmado histológicamente mediante biopsia. Las defunciones fueron confirmadas a través del Servicio de Registro Civil e Identificación de Chile. Se consideró el cierre del estudio el 31 de julio de 2007. Se construyeron las curvas de sobrevida por el método de Kaplan Meier, y estas fueron comparadas mediante el test de logrank. Se utilizó el modelo de riesgos proporcionales de Cox para el análisis multivariado. Los intervalos de confianza son de nivel $95 \%$ y las dócimas se consideraron significativas con $p<0,05$. Los datos se procesaron en el programa STATA versión 9.2

\section{RESULTADOS}

La edad promedio de las pacientes fue $52 \pm 16$ años (rango: 24 a 88 años). El tipo histológico más frecuentemente diagnosticados fueron: carcinoma $(89,2 \%)$ y adenocarcinoma $(10,7 \%)$. La edad promedio de fallecimiento por Cacu fue $55 \pm 17$ años, mientras que la edad promedio de la población sobreviviente fue $48 \pm 14$ años ( $p=0,008)$. El número total de pacientes diagnosticados con Cacu fue de 108 pacientes con una mortalidad global de $53,7 \%$. La frecuencia por estadio fue: estadio I $22,2 \%$, estadio II $21,3 \%$, estadio III $53,7 \%$ y estadio IV $2,7 \%$. La mortalidad global fue: estadio I 20,8\%, estadio II $39,1 \%$, estadio III $70,6 \%$ y estadío IV $100 \%$. La sobrevida a 3 años desde el momento del diagnóstico fue de $52 \%(I C=42,0-60,8)$ y la sobrevida a 5 años fue de $48 \%(I C=38,3-57,2)$.

La sobrevida difiere significativamente por estadio $(p=0,000)$ (Figura 1). Tomando como referencia el estadio I, las pacientes en estadio II tienen un riesgo de morir de 2,2 veces mayor $(p=0,178)$. Sin embargo, para estadio III fue 5,6 veces mayor $(p=0,000)$ y en estadio IV de $124,4(p=0,000)$. Por lo cual el factor independiente más importante en la tasa de sobrevida en Cacu es el estadio al mo- 
mento del diagnóstico, siendo significativo para el estadio III y IV (Tabla I).

En estadio I la sobrevida a 3 años fue $83 \%$ (IC:

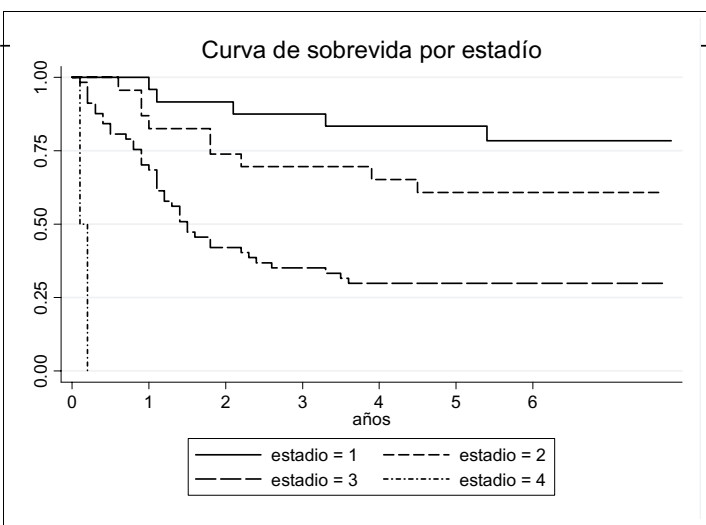

Figura 1. Cáncer cérvico uterino: sobrevida por estadio.

Tabla I

\section{RIESGO RELATIVO DE MORIR POR CÁNCER CÉRVICO UTERINO SEGÚN ESTADIO}

\begin{tabular}{cccc}
\hline Estadio & $\mathrm{HR} \pm \mathrm{DE}$ & Valor $\mathrm{p}$ & $95 \% \mathrm{IC}$ \\
\hline 1 & 1,0 & - & - \\
2 & $2,1 \pm 1,18$ & 0,1780 & $0,7-6,3$ \\
3 & $5,6 \pm 2,65$ & 0,0000 & $2,2-14,2$ \\
4 & $124,4 \pm 118,69$ & 0,0000 & $19,2-807,5$
\end{tabular}

HR: hazard ratio. DE: desvío estándar. IC: intervalo de confianza.

$61,4-93,4)$ y a los 5 años de $78 \%$ (IC=55,5-90,4). En estadio II la sobrevida a los 3 años fue $65 \%$ $(\mathrm{IC}=42,4-80,8)$ y a los 5 años de $60 \% \quad(\mathrm{IC}=38,3-$ $77,4)$. En estadio III la sobrevida a los 3 años fue $33 \%(I C=21,6-45,5)$ y a los 5 años $29 \%(I C=18,6-$ $41,9)$. En estadio IV la mortalidad fue $100 \%$ dentro de los 3 primeros meses desde su diagnóstico.

En relación a la subclasificación de los estadios, es decir estadio I a-b, estadio II a-b, estadio III a-b y estadio IV $a-b$ no hay diferencias significativas, como tampoco según el tipo histológico. Sí es predictor de sobrevida el estadio. Al ajustar los HR (hazard ratio) de los estadios por la edad se puede concluir que la edad no es un factor de riesgo significativo (Tabla II).
Tabla II

\section{HAZARD RATIO AJUSTADO (REGRESIÓN DE COX) DE CADA ESTADIO SEGÚN EDAD}

\begin{tabular}{cccc}
\hline Estadio & HR & Valor $\mathrm{p}$ & $95 \% \mathrm{IC}$ \\
\hline 1 & 1,00 & & \\
2 & 2,20 & 0,1580 & $0,74-6,57$ \\
3 & 5,57 & 0,0000 & $2,18-14,22$ \\
4 & 96,24 & 0,0000 & $14,43-641,88$ \\
edad & 1,01 & 0,1070 & $1,00-1,03$ \\
\hline
\end{tabular}

HR: hazard ratio. IC: intervalo de confianza.

\section{DISCUSIÓN}

De acuerdo a estos resultados, podemos señalar que en la Unidad de Patología Cervical del Servicio de Ginecología y Obstetricia del Hospital San José la sobrevida a 3 y 5 años es similar a las cifras reportadas en la literatura internacional en los estadios I y II. En relación a los estadios III y IV destaca una menor sobrevida a 5 años (estadio III $29 \%$ vs $37,9 \%$ y estado IV $0 \%$ vs $10,5 \%$ ) (10). En la literatura nacional no se encuentra un registro adecuado sobre la mortalidad y sobrevida a 3 y 5 años del Cacu en su diferentes estadios. Destaca un trabajo estudio nacional presentado en 2003 , donde se señala la sobrevida global de $88,9 \%$ y mortalidad de $11,1 \%$ con una mediana de seguimiento de 24 meses en una población de pacientes con cáncer Cacu estadio IB2 (11). Es importante destacar el $100 \%$ de mortalidad en estadio IV debiendo hacer énfasis en la pesquisa precoz (prevención secundaria; tamizaje con PAP) y en el oportuno y adecuado tratamiento (prevención terciaria), que son finalmente los factores que determinarán en parte el pronóstico y sobrevida de la paciente.

Otro punto importante de discusión es el hecho de que a pesar de la reducción significativa de la mortalidad por Cacu entre 1997 y 2003, fundamentalmente por el aumento en la cobertura del Papanicolaou y protocolos establecidos de manejo y tratamiento, al momento del diagnóstico, el pronóstico de sobrevida es bajo si consideramos que el $53,7 \%$ de los Cacu son diagnosticados en estadio III y la sobrevida a 3 años es de $33 \%$ y a 5 años de $29 \%$. $\mathrm{Ha}$ existido un aumento en la cobertura del PAP con detección y tratamiento precoz de lesiones premalignas, pero al momento de diagnosticar un Cacu el estadio avanzado afecta la sobrevida de la paciente. 
En relación al tipo histológico, el adenocarcinoma presenta una distribución porcentual y etaria similar a las cifras internacionales correspondiente a un $10,7 \%$ del total de los cánceres cervicouterinos y a una edad media de 47 años (12).

En Chile, en 1999, se definió un protocolo para el Tratamiento del Cáncer Invasor, incorporándose la radio - quimioterapia. Este manejo que está incorporado actualmente a las garantías explícitas en salud (GES) se basa en la recomendación de Salud Pública emitida por el Instituto Nacional del Cáncer de Estados Unidos. Esta recomendación surge de la evidencia de 5 estudios de fase 3 publicados, correspondientes a un número importante de pacientes tratadas con radioquimioterapia concomitante, empleando todos ellos cisplatino. Estos estudios demostraron reducciones similares de un 30 a 50\%, en el riesgo de muerte por Cacu, y mejoría absoluta en la sobrevida de 10 a 15\% (13-17). La evidencia surgida de estos estudios hizo que el Instituto Nacional del Cáncer de Estados Unidos (18) emitiera una recomendación de Salud Pública en Febrero de 1999 (vía correo electrónico), para incorporar la quimioterapia basada en cisplatino, concomitante con radioterapia, en el tratamiento del carcinoma invasor de cérvix localmente avanzado.

Actualmente el Cacu es una patología AUGE con garantías de acceso, oportunidad y calidad (19). Garantías de confirmación diagnóstica y tratamientos oportunos a plazos máximos, cuyo impacto aún no ha sido evaluado por ser una medida reciente, recordando que para ver los efectos posterior a la implementación de un programa deben transcurrir al menos 10 años desde su implementación.

Finalmente, debemos señalar la importancia en el diagnóstico oportuno y tratamiento óptimo para reducir la mortalidad por Cacu, mejorando la comunicación entre la redes y estableciendo sistemas de registros adecuados y actualizados.

\section{BIBLIOGRAFÍA}

1. Franco EL, Duarte-Franco ED, Ferenczy A. Cervical cancer: epidemiology, prevention and the role of human papillomavirus infection. CMAJ 2001;164(7):1017-25.

2. Donoso E, Cuello M. Mortalidad por cáncer en la mujer chilena. Análisis comparativo entre los años 1997 y 2003. Rev Chil Obstet Ginecol 2006;71(1):10-6.

3. Comisión Nacional del cáncer cérvicouterino, Ministerio de Salud. Diagnóstico y tratamiento del cáncer cérvico-uterino. Documento de trabajo, abril de 2004.

4. Donoso E, Cuello M, Villaroel L. Reducción de la mortalidad por cáncer cérvico uterino en Chile, 1990-2003. Rev Chil Obstet Ginecol 2006;71(5):307-12.

5. Suárez E, Prieto M, Rojas I, et al. Programa Nacional de Cáncer Cérvico Uterino. Rev Chil Obstet Ginecol 2001; 66(6):480-91.

6. Ministerio de Salud de Chile. Orientaciones para pesquisa y control del cáncer cérvico uterino, 1997.

7. Capurro I, Rojo JA, Pino T, Vásquez C, Garay J, Venegas $M$. Programa de detección y control de cáncer de cuello uterino en el Servicio de Salud Araucanía Sur. Rev Chil Obstet Ginecol 2002;67:114-20.

8. Eifel PJ, Morris M, Wharton JT, Oswald, MJ The influence of tumor size and morphology on the outcome of patients with FIGO stage IB squamouscell carcinoma of the uterine cervix. Int J Radiation Oncology Biol Phys 1994;29(1):9-16.

9. Perez CA, Grigsby PW, Nene SM, et al. Effect of tumor size on the prognosis of carcinoma of the uterine cervix treated with irradiation alone. Cancer 1992;69(11):2796806.

10. Todd R, Shafi M. Invasive cervical cancer. Current Obstet Gynaecol 2004;14(3):200-6.

11. San Martín $R$, Suárez $E$, Olfos $P$, et al. Cáncer cervicouterino en estadio IB2: Tratamiento con radioquimioterapia concomitante y cirugía. Rev Chil Obstet Ginecol 2003;68(6):447-57.

12. Shiliang Liu, Robert Semenciw, Yang Ma. Cervical cancer: the increasing incidence of adenocarcinoma and adenosquamous carcinoma in younger women. CMAJ 2001;164(8):1151-2.

13. Keys HM, Bundy BN, Stehman FB, et al. Cisplatin, radiation, and adjuvant hysterectomy compared with radiation and adjuvant hysterectomy for bulky stage $\mathrm{lb}$ cervical carcinoma. N Engl J Med 1999;340:1154-61.

14. Rose PG, Bundy BN, Watkins EB, et al. Concurrent cisplatin based radiotherapy and chemotherapy for locally advanced cervical cancer. NEnglJ Med 1999;340:114453.

15. Morris M, Eiffel PJ, Lu J, et al. Pelvic radiation with concurrent chemotherapy compared with pelvic and para-aortic radiation for high-risk cervical cancer. $\mathrm{N}$ Engl J Med 1999; 340:1137-43.

16. Whitney CW, Sauce W, Bundy BN, et al. A randomized comparison of fluorouracil plus cisolatin versus hydroxyurea as an adjunct to radiation therapy in stage IIB-IVA carcinoma of the cervix with negative paraaortic lymph nodes. J ClinOncol 1999;17(5):1339-48.

17. Peters W, Liu P, Barret R, et al. Cisplatin, 5-fluorouracil plus radiation therapy are superior to radiation therapy as adjunctive in high-risk, early stage carcinoma of the cervix after radical hysterectomy and pelvic lymphadenectomy: report of a phase III inter-group study. Presented at the 30th Annual Meeting of the Society of Gynecologic Oncologists, San Francisco, March 2024, 1999

18. National Cancer Institute. Concurrent chemoradiation for cervical cancer. Clinical Announcement, Washington DC, Februrary 22, 1999.

19. Gobierno de Chile, Ministerio de Salud. GuíaClínica: CáncerCérvicoUterino, 2005. Hallado en: http://webhosting.redsalud.gov.cl/minsal/archivos/guiasges/ CancerCervicouterino.pdf 